\title{
Estimating Dynamic Connectivity States in fMRI Using Regime-Switching Factor Models
}

\author{
Chee-Ming Ting*, Member, IEEE, Hernando Ombao, S. Balqis Samdin and Sh-Hussain Salleh
}

\begin{abstract}
We consider the challenges in estimating staterelated changes in brain connectivity networks with a large number of nodes. Existing studies use sliding-window analysis or time-varying coefficient models which are unable to capture both smooth and abrupt changes simultaneously, and rely on adhoc approaches to the high-dimensional estimation. To overcome these limitations, we propose a Markov-switching dynamic factor model which allows the dynamic connectivity states in functional magnetic resonance imaging (fMRI) data to be driven by lowerdimensional latent factors. We specify a regime-switching vector autoregressive (SVAR) factor process to quantity the time-varying directed connectivity. The model enables a reliable, data-adaptive estimation of change-points of connectivity regimes and the massive dependencies associated with each regime. We develop a three-step estimation procedure: 1) extracting the factors using principal component analysis, 2) identifying connectivity regimes in a low-dimensional subspace based on the factor-based SVAR model, 3) constructing high-dimensional state connectivity metrics based on the subspace estimates. Simulation results show that our estimator outperforms K-means clustering of timewindowed coefficients, providing more accurate estimate of timeevolving connectivity. It achieves percentage of reduction in mean squared error by $60 \%$ when the network dimension is comparable to the sample size. When applied to resting-state fMRI data, our method successfully identifies modular organization in restingstate networks in consistency with other studies. It further reveals changes in brain states with variations across subjects and distinct large-scale directed connectivity patterns across states.
\end{abstract}

Keywords: Regime-switching models, large VAR models, factor analysis; dynamic brain connectivity, fMRI.

\section{INTRODUCTION}

$\mathbf{F}$ UNCTIONAL connectivity (FC), the statistical dependency between distinct brain regions, is usually inferred from neuroimaging data e.g. functional magnetic resonance imaging (fMRI) time series from brain voxels [1], [2]. Most analyses assume static (stationary) FC across time, where timeinvariant FC metrics such as correlations between fMRI time series are computed over the entire period of recording. Recent years have seen increased interest in investigating dynamic changes in FC patterns over time, often referred to as $d y$ namic (time-varying) functional connectivity [3], [4]. Several studies reported temporal fluctuations in FC at time-scales of

C.-M. Ting is with the Center for Biomedical Engineering (CBE), Universiti Teknologi Malaysia (UTM), 81310 Skudai, Johor, Malaysia, and also the Computer, Electrical and Mathematical Sciences and Engineering Division, King Abdullah University of Science and Technology, Thuwal 23955, Saudi Arabia (e-mail: cmting@utm.my).

H. Ombao, is with the Computer, Electrical and Mathematical Sciences and Engineering Division, King Abdullah University of Science and Technology, Thuwal 23955, Saudi Arabia, and also the Department of Statistics, University of California at Irvine, Irvine CA 92697, USA (e-mail: hernando.ombao@kaust.edu.sa).

S. B. Samdin and S.-H. Salleh are with the Center for Biomedical Engineering (CBE), Universiti Teknologi Malaysia (UTM), 81310 Skudai, Johor, Malaysia (e-mail: sitibalqissamdin@gmail.com; hussain@fke.utm.my). seconds to minutes, in both strength and directionality of the connections, even during resting state $[5]-[8]$.

The simplest and most common approach to examining the dynamic behavior in connectivity is the sliding-window correlation, which involves computing locally stationary correlations over consecutive windowed short-time segments of data to produce time-varying FC metrics [3], [5], [6], [8]. However, this approach is limited by the choice of the optimal window length: a long window has low statistical power to detect abrupt and highly localized changes, while a short window produces noisy estimates for smooth changes. An alternative strategy is the model-based approach, which can provide a unified, parsimonious framework to characterize the dynamic connectivity structure based on the time-dependent model parameters. For example, the time-varying multivariate volatility models [9] and the time-varying vector autoregressive (VAR) models [10], [11] have been used to capture effectively instantaneous temporal changes in fMRI-based functional and effective connectivity (a more specific cross-dependence with directionality, in a sense that it measures the causal influence of one brain region on another).

Recent evidence from fMRI studies suggested state-related types of dynamics in FC: time-varying but reoccurring connectivity patterns that switch according to a few discrete underlying quasi-stable brain states (regimes) [3], [6], [12]. This non-stationarity is characterized by rapid transitions between regimes and smooth changes within a regime. Various analytical approaches have been used to identify these repeatable dynamic 'connectivity states' over time [3]. These include K-mean clustering of windowed correlations [3], [6], which, however, ignores information about the temporal order of the dynamics, hidden Markov models (HMM) producing the statetime alignments [13], or algorithms to detect change points in connectivity [12], [14]. However, these studies focused on evaluating the un-directed connectivity. Our recent work [15] proposed a more general method based on the switching VAR (SVAR) models to infer dynamic states of effective connectivity in fMRI and EEG data, but is limited to few brain regions. The next challenge is to estimate high-dimensional connectivity states for a large number of regions, where traditional analyses based on pair-wise correlations or imposing sparsity constraints might produce sub-optimal results.

In this paper, we propose a new approach based on regimeswitching factor models for estimating temporal changes in effective connectivity states in high-dimensional fMRI data for a whole-brain network analysis. Precisely, our approach uses a factor model to characterize the large fMRI data via a small number of common, latent (unobservable) factor components, and then identify the dynamic connectivity regimes based on these low-dimensional summary signals. We develop a non- 
stationary factor model which takes into account the timevariation of the underlying serial cross-correlation structure of the high-dimensional data, by introducing regime-switching in the factor dynamics. By specifying the factors to evolve as a Markov-SVAR process, we derive a factor SVAR model for the observation space. Such formulation implies projection of the high-dimensional directed connectivity matrix onto a lowerdimensional subspace (small VAR coefficient matrix of factors, spanned by the factor loadings), and thus allows us to capture the changes in connectivity regimes in the subspace driven by the few factors. It enables a reliable and computationallyefficient estimation of the regime change-points and the massive dependence measures associated with each regime. The proposed method integrates our two previous approaches with substantial enhancements: (1) It extends the SVAR model [15] by specifying the regime-switching in the lower-dimensional factor subspace rather than the observation space directly, which improves scalability for high-dimensional inference. (2) It generalizes the factor VAR model with time-invariant parameters [16] by introducing nonstationarity in the factor structure, which enables estimation of dynamic connectivity.

We develop a three-step estimation procedure. The first step is initial estimation of connectivity subspace shared across regimes based on a stationary factor model. The number of factors and a common factor loading (specifying the dimension and the span of the subspace) are estimated by applying the principal component analysis (PCA) to the data. The second step is dynamic regime segmentation based on the factor SVAR model formulated in a state-space form. The changepoints between connectivity states are identified via switching Kalman filter and switching Kalman smoother (SKF and SKS). Simultaneously, the regime-dependent parameters of the latent switching factor process are updated by the expectationmaximization (EM) algorithm, with the common factor loadings initialized and fixed using the estimates from the first step. Then, the fMRI signals are partitioned according to the estimated states, and fitted with a separate factor model for each regime to obtain regime-dependent factor loadings. The third step is estimation of within-regime connectivity metrics, where the high-dimensional VAR connectivity graph/matrix of each state is constructed from the low-dimensional factor subspace parameters estimated from the previous two steps. We evaluated the performance of our method via simulations by comparing with the K-mean clustering approach. Application to resting-state fMRI data reveals switching states of the large-scale connectivity networks with modular organization that changes across different states.

\section{REGIME-SWITCHING FACTOR MODELS}

We first describe a stationary factor model with AR factor process [16]. To overcome its limitation to static brain connectivity, we develop a nonstationary model for dynamic connectivity by allowing regime-switching in the factor dynamics.

\section{A. The Factor Model}

Let $\mathbf{y}_{t}=\left[y_{1 t}, \ldots, y_{N t}\right]^{\prime}$ be a $N \times 1$ observed vector of time series of fMRI at time points $t=1, \ldots, T$. The cross-section dimension of the time series $N$ (corresponding to the number of nodes in a brain network) can be comparable to or even larger than the length of time series $T$ (or sample size). We suppose the high-dimensional $\mathbf{y}_{t}$ is driven by a small number of latent factors. Specifically, we consider a factor model

$$
\mathbf{y}_{t}=\mathbf{Q} \mathbf{f}_{t}+\boldsymbol{\epsilon}_{t}
$$

where $\mathbf{f}_{t}=\left[f_{t 1}, \ldots, f_{t r}\right]^{\prime}$ is a $r \times 1$ vector of unobserved common factors with mean zero and covariance matrix $\boldsymbol{\Sigma}_{\mathbf{f}}$, $\mathbf{Q}=\left[\mathbf{q}_{1}, \ldots, \mathbf{q}_{r}\right]$ is a $N \times r$ constant factor loading matrix assumed to be orthonormal, i.e. $\mathbf{Q}^{\prime} \mathbf{Q}=\mathbf{I}_{r}$ where $\mathbf{I}_{r}$ denotes a $r \times r$ identity matrix, $r$ is the number of factors satisfying $r<<N$, and $\boldsymbol{\epsilon}_{t}=\left[\epsilon_{t 1}, \ldots, \epsilon_{t N}\right]^{\prime}$ is $N \times 1$ vector of noise components with mean zero and covariance matrix $\boldsymbol{\Sigma}_{\boldsymbol{\epsilon}}=\operatorname{diag}\left(\sigma_{\epsilon_{1}}^{2}, \ldots, \sigma_{\epsilon_{N}}^{2}\right)$, assuming the error terms are crosssectionally uncorrelated. The model captures the correlation between the time series via the mixing of some common factors $\mathbf{f}_{t}$ by $\mathbf{Q}$. The model (1) allows for dimension-reduction in the sense that the serial and cross-correlation in the highdimensional observational process $\left\{\mathbf{y}_{t}\right\}$ is driven by the much lower-dimensional factor process $\left\{\mathbf{f}_{t}\right\}$ and mixing matrix $\mathbf{Q}$.

The evolution of the latent factor dynamics in $\left\{\mathbf{f}_{t}\right\}$ can be modeled by a stationary VAR process of order $P, \operatorname{VAR}(P)$

$$
\mathbf{f}_{t}=\boldsymbol{\Phi}_{\mathbf{f}}(1) \mathbf{f}_{t-1}+\ldots+\boldsymbol{\Phi}_{\mathbf{f}}(P) \mathbf{f}_{t-P}+\boldsymbol{\eta}_{t}, \quad \boldsymbol{\eta}_{t} \sim N\left(\mathbf{0}, \boldsymbol{\Sigma}_{\boldsymbol{\eta}}\right)
$$

where $\mathbf{\Phi}_{\mathbf{f}}(\ell)$ is the $r \times r$ AR coefficients matrix at lag $\ell$ for $\ell=$ $1, \ldots, P$ and $\boldsymbol{\eta}_{t}$ is a $r \times 1$ Gaussian white noise process with mean zero and covariance matrix $\boldsymbol{\Sigma}_{\boldsymbol{\eta}}$. Both processes $\left\{\mathbf{y}_{t}\right\}$ and $\left\{\mathbf{f}_{t}\right\}$ are stationary where the factor loadings $\mathbf{Q}$ and the AR coefficients matrices for factors $\boldsymbol{\Phi}_{\mathbf{f}}(\ell)$ are time-constant.

1) Factor VAR Model: The temporal inter-dependence in the high-dimensional observation process $\mathbf{y}_{t}$ can be characterized by the much lower-dimensional VAR process of $\mathbf{f}_{t}$ in (2). This forms the basic idea of our recent works [16], where we developed a factor-based VAR (f-VAR) model for the observations $\mathbf{y}_{t}$ by substituting (2) into (1) and assuming $\epsilon_{t}$ approximately zero, which gives

$$
\mathbf{y}_{t}=\sum_{\ell=1}^{P} \boldsymbol{\Phi}_{\mathbf{y}}(\ell) \mathbf{y}_{t-\ell}+\mathbf{Q} \boldsymbol{\eta}_{t}
$$

where $\boldsymbol{\Phi}_{\mathbf{y}}(\ell)=\mathbf{Q} \boldsymbol{\Phi}_{\mathbf{f}}(\ell) \mathbf{Q}^{\prime}$ are high-dimensional $N \times N$ coefficients matrices for $\mathbf{y}_{t}$, an orthogonal projection onto lower-dimensional subspace spanned by the columns of $\mathbf{Q}$, with smaller matrices $\boldsymbol{\Phi}_{\mathbf{f}}(\ell)$. It provides a low-rank approximation for the dependence structure in $\mathbf{y}_{t}$. The model subspace can be learned by using the principal component analysis (PCA) where the estimator for $\mathbf{Q}$ is defined by eigenvectors corresponding to the $r$ largest eigenvalues of the sample covariance matrix of $\mathbf{y}_{t}$. The coefficients matrix $\boldsymbol{\Phi}_{\mathbf{y}}(\ell)$ can quantify directed interactions in a network with large number of nodes at time lag $\ell$. There exists a directed influence in the Granger-causality sense with direction from node $q$ to node $p$ for any connection strength $\left|\boldsymbol{\Phi}_{p q}\right|>0$, where $\boldsymbol{\Phi}_{p q}$ is the $(p, q)$-th element of $\boldsymbol{\Phi}_{\mathbf{y}}$. When applied to identify large-scale effective brain connectivity networks with a large number of brain regions from resting-state fMRI data [16], the method provides estimates with more reliable interpretation and is able to identify the modular, hierarchical structure of the brain networks during rest, by varying the subspace dimension $r$. 


\section{B. Regime-switching in Factor Dynamics}

1) Factor Model with Regime-Switching: We now generalize the stationary factor model in (1) to allow for time-variation in the serial interdependence structure of the latent factors, by introducing regime-switching in the coefficient matrices of the VAR factor process in 2. In this respect, we propose a non-stationary factor model with regime-switching factor dynamics. More precisely, we assume the factor loadings to remain stationary but allows the factors to follow a MarkovSVAR process of order $P, \operatorname{SVAR}(P)$. This class of models has been applied for modeling of econometric data [17].

The SVAR model is a collection of $K$ independent VAR models, each indexed by a hidden random indicator $S_{t}$

$$
\mathbf{f}_{t}=\sum_{\ell=1}^{P} \boldsymbol{\Phi}_{\mathbf{f}}^{\left[S_{t}\right]}(\ell) \mathbf{f}_{t-\ell}+\boldsymbol{\eta}_{t}, \quad \boldsymbol{\eta}_{t} \sim N\left(\mathbf{0}, \boldsymbol{\Sigma}_{\boldsymbol{\eta}}^{\left[S_{t}\right]}\right)
$$

here $\left\{S_{t} \in\{j=1, \ldots, K\}, t=1, \ldots, T\right\}$ is a sequence of state/regime variables, which is time-dependent and take values in a discrete space $j=1, \ldots, K$; and $\left\{\boldsymbol{\Phi}_{\mathbf{f}}^{[j]}(\ell), \ell=\right.$ $1, \ldots, P\}$ are coefficient matrices for state $j$. We assume $S_{t}$ to follow a $K$-state first-order Markov process with a $K \times K$ transition matrix $\mathbf{Z}=\left[z_{i j}\right]_{1 \leq i, j \leq K}$ where

$$
z_{i j}=P\left(S_{t}=j \mid S_{t-1}=i\right)
$$

denotes the probability of transition from state $i$ at time $t-1$ to state $j$ at $t$. Only one latent process (and hence only one VAR process) is "active" (or turned on) at each time point $t$. The remaining latent processes are turned off. This allows recurring changes in the temporal interdependence structure of the factors as characterized by $\boldsymbol{\Phi}_{\mathbf{f}}^{\left[S_{t}\right]}$, which switches over time between the finite number of regimes, according to the regime indicator $S_{t}$ at time $t$.

Remarks: Model (4) is a generalized version of (2) which allows for structural changes in the VAR coefficients. The coefficients matrices $\boldsymbol{\Phi}_{\mathbf{f}}^{\left[S_{t}\right]}(\ell)$ are piecewise constant function of the discrete state $S_{t}$, i.e. constant within time-blocks belong to a same regime but change across different regimes. This renders the factor process piecewise stationary, a special form of departure from stationarity. Moreover, compared to using a SVAR model directly on $\mathbf{y}_{t}$ as in [15], the specification of (4) allows us to detect the change-points of the high-dimensional dependence structure based on a small number of factor series. We denote the model parameters $\boldsymbol{\theta}=\left\{\boldsymbol{\theta}_{j}=\left(\boldsymbol{\Phi}_{\mathbf{f}}^{[j]}, \boldsymbol{\Sigma}_{\boldsymbol{\eta}}^{[j]}\right): j \in\right.$ $\{1, \ldots, K\}\}$ which are assumed unknown and to be estimated.

2) Factor Switching-VAR Model: We derive a highdimensional SVAR model from the non-stationary factor model with a regime-switching VAR factor process defined by (1) and (4). The regime-switching in the interdependence structure of observations $\left\{\mathbf{y}_{t}\right\}$ can be driven by that of the lower-dimensional SVAR factors in (4). Assume that $\mathbf{Q} \mathbf{f}_{t}$ is a good approximation to $\mathbf{y}_{t}$ such that $\mathbb{E}\left\|\boldsymbol{\epsilon}_{t}\right\|_{2}^{2}=\mathbb{E}\left\|\mathbf{y}_{t}-\mathbf{Q f}_{t}\right\|_{2}^{2}$ is negligible for all $t$, substituting (4) into (1) gives

$$
\begin{aligned}
\mathbf{y}_{t} & =\sum_{\ell=1}^{P} \mathbf{Q} \boldsymbol{\Phi}_{\mathbf{f}}^{\left[S_{t}\right]}(\ell) \mathbf{Q}^{\prime} \mathbf{Q} \mathbf{f}_{t-\ell}+\mathbf{Q} \boldsymbol{\eta}_{t} \\
& =\sum_{\ell=1}^{P} \mathbf{Q} \boldsymbol{\Phi}_{\mathbf{f}}^{\left[S_{t}\right]}(\ell) \mathbf{Q}^{\prime} \mathbf{y}_{t-\ell}+\mathbf{Q} \boldsymbol{\eta}_{t} .
\end{aligned}
$$

Then, we have a factor-based SVAR (f-SVAR) model for $\mathbf{y}_{t}$

$$
\mathbf{y}_{t}=\sum_{\ell=1}^{P} \boldsymbol{\Phi}_{\mathbf{y}}^{\left[S_{t}\right]}(\ell) \mathbf{y}_{t-\ell}+\boldsymbol{\nu}_{t}
$$

where $\boldsymbol{\Phi}_{\mathbf{y}}^{\left[S_{t}\right]}(\ell)=\mathbf{Q} \boldsymbol{\Phi}_{\mathbf{f}}^{\left[S_{t}\right]}(\ell) \mathbf{Q}^{\prime}$ and $\boldsymbol{\nu}_{t}=\mathbf{Q} \boldsymbol{\eta}_{t}$.

Remarks: The model is a nonstationary generalization of the factor VAR model (3), by allowing a regime-switching in the coefficient parameters. It provides a tool to capture regimeswitching in the large-dimensional serial inter-dependence structure in $\mathbf{y}_{t}$ via a low-dimensional space of $\mathbf{f}_{t}$. The model quantifies dynamics of a large-scale directed network with state-dependent changes in the network structure, i.e. switching according to distinct states. It enables detection of temporal change points in the network dependency structure via $S_{t}$, and characterization of the directed dependencies between massive number of nodes associated with each state by $\boldsymbol{\Phi}_{\mathbf{y}}^{[j]}$.

3) State-Space Formulation: We propose a state-space representation for the factor model with regime-switching factors, to enable sequential estimation in time of the latent factors $\left\{\mathbf{f}_{t}\right\}$ and the switching states $\left\{S_{t}\right\}$. The latent SVAR factor process (4) forms the state equation which is projected to the high-dimensional space using the factor model (1) with an additive error in the observation equation. Defining the dynamic factor structure $\mathbf{F}_{t}=\left[\mathbf{f}_{t}^{\prime}, \mathbf{f}_{t-1}^{\prime}, \ldots, \mathbf{f}_{t-P+1}^{\prime}\right]^{\prime}$ as state vector, the model (1) and (4) are formulated in a switching linear Gaussian state-space form [18]

$$
\begin{aligned}
& \mathbf{F}_{t}=\mathbf{A}_{\mathbf{F}}^{\left[S_{t}\right]} \mathbf{F}_{t-1}+\mathbf{w}_{t} \\
& \mathbf{y}_{t}=\mathbf{H F}_{t}+\boldsymbol{\epsilon}_{t}
\end{aligned}
$$

The $\operatorname{SVAR}(P)$ factor process (4) is re-written in a $\operatorname{SVAR}(1)$ form of $[8]$ in the state equation, where $\mathbf{w}_{t}=\left[\boldsymbol{\eta}_{t}^{\prime}, \mathbf{0}^{\prime}, \ldots, \mathbf{0}^{\prime}\right]^{\prime}$ is $r P \times 1$ state noise, and $\mathbf{A}_{\mathbf{F}}^{\left[S_{t}\right]}$ is a $r P \times r P$ state transition matrix switching with the state variables $S_{t}$, and of the form

$$
\mathbf{A}_{\mathbf{F}}^{\left[S_{t}\right]}=\left(\begin{array}{ccccc}
\boldsymbol{\Phi}_{\mathbf{f}}^{\left[S_{t}\right]}(1) & \boldsymbol{\Phi}_{\mathbf{f}}^{\left[S_{t}\right]}(2) & \ldots & \boldsymbol{\Phi}_{\mathbf{f}}^{\left[S_{t}\right]}(P-1) & \boldsymbol{\Phi}_{\mathbf{f}}^{\left[S_{t}\right]}(P) \\
\mathbf{I}_{r} & \mathbf{0} & \ldots & \mathbf{0} & \mathbf{0} \\
\mathbf{0} & \mathbf{I}_{r} & \ldots & \mathbf{0} & \mathbf{0} \\
\vdots & & \ddots & & \vdots \\
\mathbf{0} & \mathbf{0} & \ldots & \mathbf{I}_{r} & \mathbf{0}
\end{array}\right)
$$

The matrix $\mathbf{A}_{\mathbf{F}}^{\left[S_{t}\right]}$ describes the directed connectivity that varies across states. The unobserved $\operatorname{SVAR}(P)$ dynamic factors $\mathbf{F}_{t}$ now follows a latent SVAR(1) process. The factor model (1) is re-formulated as the observation equation (9) with the idiosyncratic noise $\boldsymbol{\epsilon}_{t}$, and a $N \times r P$ mapping matrix $\mathbf{H}=[\mathbf{Q}, \mathbf{0}, \ldots, \mathbf{0}]$. We assume both $\left\{\boldsymbol{\epsilon}_{t}\right\}$ and $\left\{\mathbf{w}_{t}\right\}$ are white Gaussian noise, $\boldsymbol{\epsilon}_{t} \sim N\left(\mathbf{0}, \boldsymbol{\Sigma}_{\boldsymbol{\epsilon}}\right)$ and $\mathbf{w}_{t} \sim N\left(\mathbf{0}, \boldsymbol{\Sigma}_{\mathbf{w}}^{\left[S_{t}\right]}\right)$, with a time-varying state noise covariance matrices $\boldsymbol{\Sigma}_{\mathbf{w}}^{\left[S_{t}\right]}$ switching with $S_{t}$. Both the factor loadings $\mathbf{Q}$ and the noise covariance matrix $\Sigma_{\epsilon}$ in the observation equation are assumed to be regime-invariant and shared across regimes. The processes $\left\{\mathbf{f}_{t}\right\}$ and $\left\{\boldsymbol{\epsilon}_{t}\right\}$ are uncorrelated. Instead of hard state assignment for each time-point $t$, we can evaluate the probability of activation for each state, $P\left(S_{t}=j \mid \mathbf{y}_{1: T}\right)$, which is termed "soft-alignment". We denote all model parameters from each of the states as $\boldsymbol{\Theta}=\left\{\boldsymbol{\Theta}_{j}=\left(\mathbf{A}_{\mathbf{F}}^{[j]}, \boldsymbol{\Sigma}_{\mathbf{w}}^{[j]}\right): j \in\{1, \ldots, K\}\right\}$. 


\section{ESTIMATION}

We develop a three-step procedure for efficiently estimating the dynamic connectivity states in high-dimensional fMRI data based on the proposed non-stationary factor model with regime-switching. In first step, we explore the connectivity subspace assumed to be common and shared across regimes, by fitting a stationary factor model (1) to the entire fMRI time series. We apply the method of PCA to estimate the factor loadings $\mathbf{Q}$ and the latent factors $\mathbf{f}_{t}$, and the Bayesian information criterion (BIC) of [19] to select the optimal number of factors. In second step, we perform connectivity regime segmentation in the low-dimensional subspace relying on a factor model with Markov-SVAR factor process (4). Based on the state-space representation (8)-(9), the latent factor process can be jointly estimated conditioned on the observational model. The temporal change-points of the regimes can be detected via the estimated state sequence $\left\{\widehat{S}_{t}\right\}$ by the SKF and SKS, and the factor VAR coefficient matrix $\boldsymbol{\Phi}_{\mathbf{f}}^{[j]}$ for each regime is updated iteratively using the EM algorithm. In third step, we estimate the regime-dependent high-dimensional connectivity matrix $\boldsymbol{\Phi}_{\mathbf{y}}^{[j]}$ in 77 for the observation space using the estimated subspace parameters from the first two steps.

\section{A. Step 1: Estimation of a Common Factor Model}

PCA is a common approach to estimating factor model based on the eigen-decomposition of sample covariance matrix [20], [21]. Let $\mathbf{V}_{1}, \ldots, \mathbf{V}_{N}$ be $N$ orthonormal eigenvectors corresponding to the eignevalues of the $N \times N$ sample covariance matrix $\mathbf{S}_{\mathbf{y}}=T^{-1} \sum_{t=1}^{T} \mathbf{y}_{t} \mathbf{y}_{t}^{\prime}$, in a decreasing order such that $\widehat{\lambda}_{1} \geq \ldots \geq \widehat{\lambda}_{N}>0$. The PCA estimator of the loadings $\widehat{\mathbf{Q}}=\left[\mathbf{V}_{1}, \ldots, \mathbf{V}_{r}\right]$ is defined by a matrix whose columns are the $r$ orthonormal eigenvectors corresponding to the largest $r$ eignevalues, and the factors can be estimated by $\widehat{\mathbf{f}}_{t}=\widehat{\mathbf{Q}}^{\prime} \mathbf{y}_{t}$. [20] has showed that the PCA estimators are consistent and asymptotically normal, under settings of large $N$ and large $T$. Besides, the estimates can be computed efficiently even under situations when $N<T$ (on the small $T \times T$ temporal covariance matrix instead of the huge $N \times N$ spatial sample covariance $\mathbf{S}_{\mathbf{y}}$ ). We can compute the noise covariance estimator as $\widehat{\boldsymbol{\Sigma}}_{\boldsymbol{\epsilon}}=T^{-1} \sum_{t=1}^{T} \widehat{\boldsymbol{\epsilon}}_{t} \widehat{\boldsymbol{\epsilon}}_{t}^{\prime}$ based on the residuals $\widehat{\boldsymbol{\epsilon}}_{t}=\mathbf{y}_{t}-\widehat{\mathbf{Q}}_{t}$ from the fitted factor model. We fit an VAR model 2 2 to the estimated factors $\left\{\widehat{\mathbf{f}}_{t}\right\}$ by the leastsquares (LS) method, and obtain the AR coefficient estimates $\widehat{\boldsymbol{\Phi}}_{\mathbf{f}}(\ell)$. For PCA estimation, the number of factors can be determined by model selection using BIC

$\hat{r}=\underset{\left\{1, \ldots, L_{r}\right\}}{\arg \max }\left\{\ln \left(\frac{1}{N T} \sum_{t=1}^{T}\left\|\widehat{\boldsymbol{\epsilon}}_{t}(r)\right\|_{2}^{2}\right)+r\left(\frac{N+T}{N T}\right) \ln (\right.$

where $\|\mathbf{x}\|_{2}$ denotes the Euclidean norm of a vector $\mathbf{x}$ and $L_{r}$ is a bounded integer such that $r \leq L_{r}$.

\section{B. Step 2: Estimation of Regime-switching Factor Model}

Based on the state-space formulation (8)-(9), the objective is to extract the underlying states $\left\{\widehat{S}_{t}\right\}$, and to estimate the unknown coefficient matrix $\boldsymbol{\Phi}_{\mathbf{f}}^{[j]}$ and factor signals in $\mathbf{F}_{t}=\left[\mathbf{f}_{t}^{\prime}, \mathbf{f}_{t-1}^{\prime}, \ldots, \mathbf{f}_{t-P+1}^{\prime}\right]^{\prime}$ of the latent Markov-SVAR factor process given observations $\mathbf{y}_{t}, t=1, \ldots T$.
1) Choice of Number of States $K$ : In practice, the number of connectivity states $K$ in brain activity is typically unknown $a$ priori, and has to be estimated first. It can be determined by a data-driven approach via estimating the number of clusters in time-variant VAR (TV-VAR) coefficients, using some cluster validity indexes e.g. the variance ratio criterion, as in [15].

2) Filtering and Smoothing: The inference of $S_{t}$ and $\mathbf{F}_{t}$ involve computing, sequentially in time, the filtered probabilities $\operatorname{Pr}\left(S_{t} \mid \mathbf{y}_{1: t}\right)$ and the filtered densities $p\left(\mathbf{F}_{t} \mid \mathbf{y}_{1: t}\right)$, given the available signal observations up to time $t, \mathbf{y}_{1: t}=\left\{\mathbf{y}_{1}, \ldots, \mathbf{y}_{t}\right\}$, and the more accurate smoothed probabilities $P\left(S_{t} \mid \mathbf{y}_{1: T}\right)$ and densities $p\left(\mathbf{F}_{t} \mid \mathbf{y}_{1: T}\right)$ given the available entire set of observations $\mathbf{y}_{1: T}=\left\{\mathbf{y}_{1}, \ldots, \mathbf{y}_{T}\right\}$. We estimate the filtered and smoothed densities of $\mathbf{F}_{t}$ given state $j$ at time $t$, by the $\mathrm{KF}$ and the KS, respectively

$$
\begin{aligned}
\mathbf{F}_{t \mid t}^{j} & =\mathbb{E}\left(\mathbf{F}_{t} \mid \mathbf{y}_{1: t}, S_{t}=j\right) \\
V_{t \mid t}^{j} & =\mathbb{C o v}\left(\mathbf{F}_{t} \mid \mathbf{y}_{1: t}, S_{t}=j\right) \\
\mathbf{F}_{t \mid T}^{j} & =\mathbb{E}\left(\mathbf{F}_{t} \mid \mathbf{y}_{1: T}, S_{t}=j\right) \\
V_{t \mid T}^{j} & =\operatorname{Cov}\left(\mathbf{F}_{t} \mid \mathbf{y}_{1: T}, S_{t}=j\right) \\
V_{t, t-1 \mid T}^{j} & =\operatorname{Cov}\left(\mathbf{F}_{t}, \mathbf{F}_{t-1} \mid \mathbf{y}_{1: T}, S_{t}=j\right)
\end{aligned}
$$

where $\mathbf{F}_{t \mid t}^{j}$ and $V_{t \mid t}^{j}$ are mean and covariance of the filtered density $p\left(\mathbf{F}_{t} \mid \mathbf{y}_{1: t}, S_{t}=j\right), \mathbf{F}_{t \mid T}^{j}$ and $V_{t \mid T}^{j}$ are mean and covariance of the smoothed density $p\left(\mathbf{F}_{t} \mid \mathbf{y}_{1: T}, S_{t}=j\right)$ given state $j$ at time $t$, and $V_{t, t-1 \mid T}^{j}$ is the cross-variance of joint density $p\left(\mathbf{F}_{t}, \mathbf{F}_{t-1} \mid \mathbf{y}_{1: T}, S_{t}=j\right)$. We also compute the estimates of filtered and smoothed state occupancy probability of being state $j$ at time $t$

$$
\begin{aligned}
\mathbf{M}_{t \mid t}^{j} & =P\left(S_{t}=j \mid \mathbf{y}_{1: t}\right) \\
\mathbf{M}_{t \mid T}^{j} & =P\left(S_{t}=j \mid \mathbf{y}_{1: T}\right)
\end{aligned}
$$

3) EM Estimation: The estimates of the factor-subspace dynamic parameters in $\mathbf{A}_{\mathbf{F}}^{[j]}$ and $\boldsymbol{\Sigma}_{\mathbf{w}}^{[j]}$ can be obtained by the maximum likelihood (ML) method by maximizing the loglikelihood $L=\log p\left(\mathbf{y}_{1: T} \mid \boldsymbol{\Theta}\right)$ with respect to each parameter. Here, we use the EM algorithm for the switching state-space model suggested by [22]. In the expectation step (E-step), the sufficient statistics are obtained from the smoothed estimates

$$
\begin{aligned}
P_{t} & =\mathbb{E}\left(\mathbf{F}_{t} \mathbf{F}_{t}^{\prime} \mid \mathbf{y}_{1: T}\right)=V_{t \mid T}+\mathbf{F}_{t \mid T} \mathbf{F}_{t \mid T}^{\prime} \\
P_{t, t-1} & =\mathbb{E}\left(\mathbf{F}_{t} \mathbf{F}_{t-1}^{\prime} \mid \mathbf{y}_{1: T}\right)=V_{t, t-1 \mid T}+\mathbf{F}_{t \mid T} \mathbf{F}_{t-1 \mid}^{\prime}
\end{aligned}
$$

where $\mathbf{F}_{t \mid T}, V_{t \mid T}$ and $V_{t, t-1 \mid T}$ are quantities of the smoothed NTdensities $p\left(\mathbf{F}_{t} \mid \mathbf{y}_{1: T}\right)$ and $p\left(\mathbf{F}_{t}, \mathbf{F}_{t-1} \mid \mathbf{y}_{1: T}\right)$, corresponding to + [73 to 15 by marginalizing out the state variable $j$ of the $p\left(\mathbf{F}_{t} \mid \mathbf{y}_{1: T}, S_{t}=j\right)$ and $p\left(\mathbf{F}_{t}, \mathbf{F}_{t-1} \mid \mathbf{y}_{1: T}, S_{t}=j\right)$ using Gaussian approximation. We retain the terms switching KF (SKF) and switching KS (SKS) to refer to $\mathrm{KF} / \mathrm{KS}$ approach to estimating state parameters of the SVAR model, as in [22].

Suppose we observe $S$ independent replicates of signals $\mathbf{y}_{s, 1: T}, s=1, \ldots S$ (from multiple subjects or trials of experiment). To fit a common model based on these replicates, we first run the E-step to compute the expectations for each replicate $s$. Then in maximization step (M-step), the parameter estimates for each regime $j$ that are common across replicates, 
are updated by accumulating the statistics over all replicates

$$
\begin{aligned}
& \widehat{\mathbf{A}}_{\mathbf{F}}^{[j]}=\left(\sum_{s=1}^{S} \sum_{t=2}^{T} W_{s t}^{j} P_{s t, t-1}\right)\left(\sum_{s=1}^{S} \sum_{t=2}^{T} W_{s t}^{j} P_{s t-1}\right)^{-1}(20) \\
& \boldsymbol{\Sigma}_{\mathbf{w}}^{[j]}=\left(\frac{1}{\sum_{s=1}^{S} \sum_{t=2}^{T} W_{s t}^{j}}\right)\left(\sum_{s=1}^{S} \sum_{t=2}^{T} W_{s t}^{j} P_{s t}\right. \\
&\left.-\widehat{\mathbf{A}}_{\mathbf{F}}^{[j]} \sum_{s=1}^{S} \sum_{t=2}^{T} W_{s t}^{j} P_{s t, t-1}^{\prime}\right)(21) \\
& \widehat{z}_{i j}=\frac{\sum_{s=1}^{S} \sum_{t=2}^{T} P\left(S_{s t-1}=j, S_{s t}=i \mid \mathbf{y}_{s, 1: T}\right)}{\sum_{s=1}^{S} \sum_{t=1}^{T-1} W_{s t}^{j}}
\end{aligned}
$$

with the weights $W_{s t}^{j}=\mathbf{M}_{s, t \mid T}^{j}$ computed from the smoothing step for each $s$. The model parameters are updated iteratively until some convergence criteria are satisfied, to produce the ML estimates $\Theta^{*}$. The initial estimate of $\widehat{\mathbf{A}}_{\mathbf{F}}^{[j]}$ is obtained by fitting segmented regimes by $\mathrm{K}$-means clustering. The factor loading matrix $\mathbf{Q}$ in $\mathbf{H}$ and the noise covariance $\boldsymbol{\Sigma}_{\boldsymbol{\epsilon}}$ (common to all regimes) are fixed with the PCA estimates from Step 1, and not updated by EM algorithm. Note that here the regime estimation is done based on the state equation of lowdimensional factors, instead of the high-dimensional latent signals in [15]. This will lead to substantial computational reduction and better estimates of the subspace parameters.

4) Regime Segmentation: Given the EM-estimated model parameters $\Theta^{*}$, the preliminary temporal regime segmentation $\widehat{\boldsymbol{\Phi}}_{\mathbf{f}}^{\left[\widehat{S}_{t}\right]}$ in the subspace is defined by the latent state sequence estimated by the SKF, $\widehat{S}_{t}^{\mathrm{SKF}}=\arg \max _{j} P\left(S_{t}=j \mid \mathbf{y}_{1: t}\right)$ in (16) which indicates the most likely active state for each time point. This is then further refined by the SKS, $\widehat{S}_{t}^{\text {SKS }}=$ $\arg \max _{j} P\left(S_{t}=j \mid \mathbf{y}_{1: T}\right)$ in 177 based on both the past and future observations. We can also utilize this state-time alignment provided in $\widehat{S}_{t}^{\mathrm{SKF}}$ and $\widehat{S}_{t}^{\mathrm{SKS}}$ to partition the observed fMRI signals into their corresponding states, and the timesegments of each regime $\Pi_{j}=\left\{\mathbf{y}_{t}: \widehat{S}_{t}^{\text {SKS }}=j\right\}, j=1, \ldots, K$ is then fitted with a separate stationary factor model to derive state-dependent estimators, as described in the next step.

\section{Step 3: Estimation of Regime Connectivity Matrices}

We investigate two different schemes for constructing the estimators for the high-dimensional VAR-based connectivity matrix or graph $\boldsymbol{\Phi}_{\mathbf{y}}^{[j]}$ for each state, by plugging in the subspace parameter estimators obtained in the first two steps: (1) Coupled SVAR estimator, with common factor loadings (fSVAR-ComQ): $\widehat{\boldsymbol{\Phi}}_{\mathbf{y}}^{[j]}(\ell)=\widehat{\mathbf{Q}} \widehat{\boldsymbol{\Phi}}_{\mathbf{f}}^{[j] *}(\ell) \widehat{\mathbf{Q}}^{\prime}$ by plugging into the f-SVAR model 77 with the EM estimate $\widehat{\boldsymbol{\Phi}}_{\mathbf{f}}^{[j] *}(\ell)$ from Step 2 and the PCA estimate $\widehat{\mathbf{Q}}$ from Step 1. Note that conditioned on a common factor loading $\widehat{\mathbf{Q}}$, the factor coefficient matrices $\widehat{\Phi}_{\mathbf{f}}^{[j] *}(\ell)$ of all regimes are jointly estimated by the EM, weighted at each state by the smoothed state occupancy probability $P\left(S_{t}=j \mid \mathbf{y}_{1: T}\right)$ in (17). (2) Decoupled SVAR estimator, with state-dependent factor loadings (f-SVAR-DepQ): $\widetilde{\boldsymbol{\Phi}}_{\mathbf{y}}^{[j]}(\ell)=\widetilde{\mathbf{Q}}^{[j]} \widetilde{\boldsymbol{\Phi}}_{\mathbf{f}}^{[j]}(\ell) \widetilde{\mathbf{Q}}^{\prime[j]}$ based on a separate f-VAR model (3) for each state, with plugged-in PCA estimators $\left(\widetilde{\mathbf{f}}_{t}^{[j]}\right.$, $\mathbf{Q}^{j j]}$ ) obtained by fitting a distinct stationary factor model (1) separately to each regime time-course $\Pi_{j}$ derived from the SKS segmentation in Step 2, and $\widetilde{\boldsymbol{\Phi}}_{\mathbf{f}}^{[j]}$ is the LS estimator based on $\left\{\widetilde{\mathbf{f}}_{t}^{[j]}\right\}$. Compared to the f-VAR estimator in [16], this is more general by using regime-dependent PCA to estimate localized model subspace specific to each state. We establish asymptotic normality of the decoupled estimator and derive a procedure for significance testing of $\widetilde{\boldsymbol{\Phi}}_{\mathbf{y}}^{[j]}$ (See Appendix).

\section{Simulations}

In this section, we evaluate the performance of the f-SVAR model-based approach for identifying state-driven changes in large-scale directed connectivity via a series of simulations. The objective is to measure the ability of the proposed estimation procedures in (1.) identifying the temporal segmentation of regimes via the estimated state sequence, and (2) estimating the high-dimensional time-evolving directed connectivity matrices or graphs. We assess the sensitivity of performance in various scenarios: the choice of number of factors, increased network dimension and number of states.

1) Data Generation: Data was simulated from a regimeswitching VAR(1) model with $K$ states, with a unique VAR coefficient matrix for each state to characterize distinct statespecific connectivity patterns. To emulate a modular connectivity network with $M$ equally-sized sub-networks of $n$ nodes, we constructed a block-diagonal coefficient matrix with $M$ diagonal sub-blocks, each with dimension $n \times n$. The dimension of entire network is $N=M n$ (i.e., the number of simulated time series). All entries of the diagonal sub-blocks are nonzero representing dense directed connections between nodes within a module. We randomly draw the values of connectivity strength from a uniform distribution $\boldsymbol{\Phi}_{\mathbf{y}}^{[j]}: a_{p q} \sim \mathrm{U}\left[-\alpha_{j} \alpha_{j}\right]$ when nodes $p$ and $q$ belong to the same sub-block. Entries of off-diagonal blocks are zero. We consider five distinct states with increasing connectivity strength by setting for each state, respectively, $\alpha_{1}=0.2, \alpha_{2}=0.4, \alpha_{3}=0.6, \alpha_{4}=0.8$, $\alpha_{5}=1.0$. We set noise covariance $\boldsymbol{\Sigma}_{\boldsymbol{\eta}}=0.1 \mathrm{I}$ for all states.

To create piece-wise stable connectivity structure over time, we concatenated $K$ time-blocks each of fixed length $T_{B}$, each simulated independently from a state-specific stationary VAR(1) model with the $\boldsymbol{\Phi}_{\mathbf{y}}^{[j]}$ generated above, according to order $j=1, \ldots, K$. Following [23], the $K$ successive timeblocks were repeated for two cycles to emulate the recurring connectivity states. Thus, the state labels and the corresponding state-dependent VAR coefficient matrices $\left(S_{t}, \boldsymbol{\Phi}_{\mathbf{y}}^{\left[S_{t}\right]}\right)$ for each time point are considered known and used as ground-truth for evaluation. The total number of time points is $T=2 K T_{B}$. The simulations were repeated 200 times.

2) Benchmark with K-means Clustering: We computed factor-SVAR model-based estimates for the state sequence $\widehat{S}_{t}, t=1, \ldots, T$ and the coefficient matrix for each state $\widehat{\boldsymbol{\Phi}}_{\mathbf{y}}^{[j]}$, using the estimation steps in Section III. We compare the performance of our estimator with the commonly used approach to estimating dynamic connectivity states, based on K-means clustering of time-varying connectivity metrics [6]. First, the sliding window technique was used to estimate the time-evolving directed connectivity, by fitting locally stationary VAR models to shifted short-time windows of fixed length to obtain the VAR coefficient matrices for each time point. We chose a rectangular window of 30 samples and 
shift of 1 sample. Then, the K-means clustering algorithm was applied to the estimated time-variant VAR (TV-VAR) coefficients (called TV-VAR-KM) to partition the dynamic connectivity structure into the distinct states or regimes. As in [6], we used the L1 (Manhattan) distance which may be more effective for clustering high-dimensional data, compared to the L2 (Euclidean) distance. Finally, the state connectivity matrices were computed by fitting independent VARs to the segmented regimes. The relatively small sample size available to each regime may render the traditional ordinary leastsquares (LS) fits of large-dimensional VAR matrices inaccurate, due to insufficient information to estimate the huge number of parameters. Therefore, we used the $L_{2}$-regularized or ridge estimator which imposes a $L_{2}$ norm penalty on the AR coefficients, to obtain a better-conditioned estimate particularly in high-dimensional settings. The regularization parameter was set $\lambda=0.1$, as suggested by [24] for VAR model estimation.

Our proposed SVAR approach has more advantages than the K-means clustering of time-variant VAR coefficients, as discussed in [15]. First, our approach provides a unified parametric framework for estimating both the dynamic states and connectivity graphs simultaneously, rather than the separate steps of sliding-window connectivity analysis followed by regime clustering. Second, the sliding-window approach is limited by the choice of window size which is crucial: a large window leads to low statistical power for detecting abrupt and highly localized changes; a small window produces noisy estimates for smooth changes. In contrast, the SVAR model is capable of detecting changes at different time scales, both smooth and abrupt, avoiding the problems associated with fixed time windowing. This is because both the long stable periods and short-time abrupt alternations between states, can be regulated via the transition probabilities of Markov chain and estimated in a sequential way at each time by the KF. Third, the K-means algorithm provides a 'hard' assignment of time points into states and does not account for the temporal correlation structure. In contrast, the SVAR estimator generates 'soft' state-time alignment by estimating sequentially, for each time point, the probability of the occupying states based on the entire observation time course.

3) Performance Measures: To quantify the performance of different methods in connectivity regime segmentation, we computed the Rand index (RI) [25] which measures the similarity between the estimated and the true partitions of the $T$ observations into states, based on $\widehat{S}_{t}$ and $S_{t}$. It is defined in terms of numbers of pairs of time points that are correctly identified as belonging to the same or different states, where RI $=1$ indicates perfect recovery of the true partition. This indirectly evaluates the change-point detection in the connectivity structure. To measure the ability to track the dynamic changes in connectivity graphs over time, we computed the mean squared error between the estimated and ground-truth time-evolving VAR coefficient matrices over the entire time course, MSE $=T^{-1} \sum_{t=1}^{T}\left\|\widehat{\boldsymbol{\Phi}}_{\mathbf{y}}^{\left[\widehat{S}_{t}\right]}-\boldsymbol{\Phi}_{\mathbf{y}}^{\left[S_{t}\right]}\right\|_{F}^{2}$ where $\|\mathbf{H}\|_{F}=\operatorname{tr}\left(\mathbf{H}^{\prime} \mathbf{H}\right)^{1 / 2}$ denotes Frobenius norm of matrix $\mathbf{H}$. We report results in median and median absolute deviation (MAD) of RIs and MSEs over 200 replications of simulation.
4) Choice of Number of Factors: In this simulation, we first investigate sensitivity of the f-SVAR estimators to the choice of $r$. Fig. 1 plots the performance in terms of RIs and MSEs against increasing $r$ from 1 to 10 . We fix $K=2, N=50$ ( $M=5, n=10)$ and $T=200$. Fig. 1 (a) shows that the regime segmentation accuracy of both the SKF and SKS $\left(\widehat{S}_{t}^{\text {SKF }}\right.$ and $\widehat{S}_{t}^{\text {SKS }}$ ) improves when using more factors, which can better explain the variability of dynamics between regimes. However, inclusion of additional factors after $r=5$ shows no significant improvement as most of the variations in signals have been sufficiently captured by the first five dominant factors. From Fig. 1.b) it is clear that the MSE of the coupled estimator (common $\mathbf{Q}$ ) remains stable initially, but starts to increase with $r$ when $r>6$. On the other hand, estimation accuracy of the decoupled estimator (regime-dependent $\mathbf{Q}$ ) improves steadily as $r$ increase until a limit at $r \geq 7$. The coupled estimator performs poorly because the component estimator, i.e. the $r \times r$ factor coefficient matrix $\widehat{\boldsymbol{\Phi}}_{\mathbf{f}}^{[j]}$ becomes less accurate for large dimensions $r$. However, this is offset by a gain in performance by using regime-specific factor loadings in the decoupled estimator, which can better capture the difference in connectivity structure between the two states, probably characterized by a large break in the loadings not only in the factor dynamics. Together with the improved regime partitioning, this results in a decrease in MSE. Therefore, misspecifying $r$ could lead to poor performance of the f-SVAR estimator and unnecessary computational effort. An appropriate data-driven approach is needed to select an optimal value of $r$.

We performed additional simulations to evaluate the ability of BIC in selecting the true number of factors under the f-SVAR model. The BIC has been shown appropriate for estimating $r$ in high-dimensional factor models with both asymptotic efficiency and good finite sample performance [19]. We followed simulations in [19] but used an SVAR as the factor generating process. We generated factors $\mathbf{f}_{t}$ from a $3 \times 1$ SVAR(1) process of (4) with independent noise $\boldsymbol{\eta}_{t} \sim N(\mathbf{0}, \mathbf{I})$. The number of states $K$ was varied from 2 to 5 , and the factor coefficient matrix was simulated $\boldsymbol{\Phi}_{\mathbf{f}}^{[j]}: a_{p q}^{f} \sim \mathrm{U}\left[-\beta_{j} \beta_{j}\right]$ with increasing strength $\beta_{j} \in\{0.2,0.4,0.6,0.8,1\}$ for each state $j=1, \ldots, 5$. In the observation model (1), the $N \times r$ elements of $\mathbf{Q}$ were generated independently from $\mathrm{U}[-0.60 .6]$. We let $N$ to increase from 20 to 100 and fix $T=200$. Table $[$ reports the frequencies of selecting the correct $r, P(\hat{r}=3)$ by the BIC in (10) over 200 replications, for increasing $N$ and $K$. The maximum number of factors evaluated is set $L_{r}=10$. We can see that the estimation for $r$ improves as the dimension $N$ increases, achieving perfect identification at $N \geq 60$ for all $K$. This is due to the behavior of 'blessing of dimensionality', making use of the increased information on the factors from the added components of $\mathbf{y}_{t}$ when $N$ increases.

5) Effect of Varying Network Dimension: In this simulation, we study the impact of network size $N$ on the dynamic connectivity estimation. We fix the sample size $T=200$ and increase the dimension $N$ from 10 to 100 (number of subnetworks $M$ from 1 to 10 with $n=10$ ) to create different scenarios of dimensionality $N<T$ and $N \approx T$. The number of factors $r$ was selected adaptively for each $N$ using BIC. Fig. 2 plots the median RIs and MSEs for the K-means 


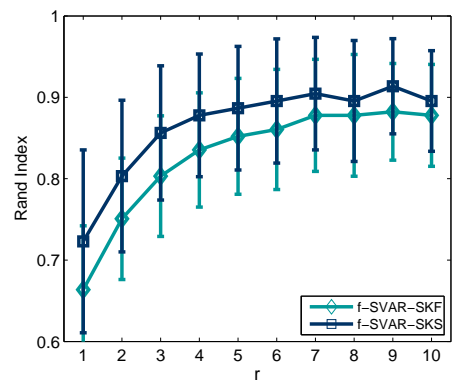

(a)

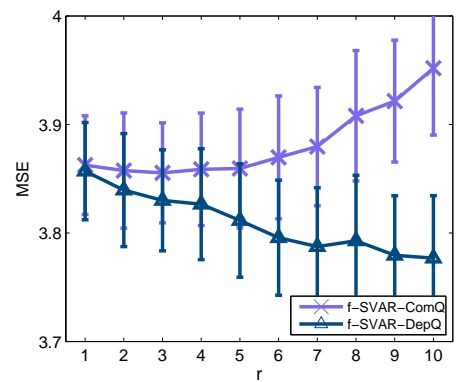

(b)

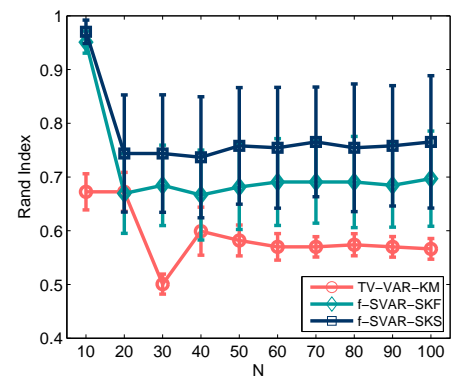

(a)

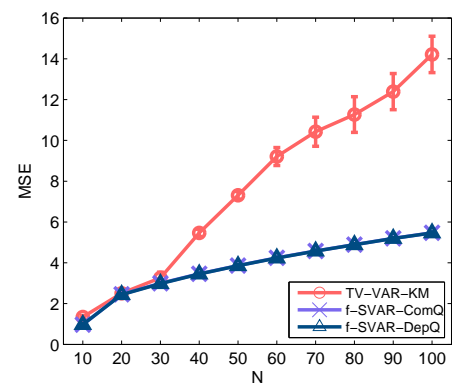

(b)

Fig. 1. Effect of number of factors $r$ on connectivity state estimation performance of f-SVAR estimators when $K=2$ and $T=200$. (a) Rand index of state segmentation by f-SVAR-SKF and f-SVAR-SKS (SKF and SKS based on f-SVAR model). (b) MSE of dynamic connectivity matrices by fSVAR-ComQ and f-SVAR-DepQ. Lines and error bars represent medians and median absolute deviations over 200 replications.

\section{TABLE I}

RELATIVE FREQUENCY OF CORRECTLY ESTIMATED NUMBER OF FACTORS $P(\hat{r}=3)$ BY BIC OVER 200 REPLICATIONS OF SIMULATION FOR DIFFERENT DIMENSIONALITY $N$ AND NUMBER OF STATES $K$.

\begin{tabular}{cccccccccc}
\hline \hline \multirow{2}{*}{$K$} & \multicolumn{10}{c}{$N$} \\
\cline { 2 - 10 } & 20 & 30 & 40 & 50 & 60 & 70 & 80 & 90 & 100 \\
\hline 2 & 0.29 & 0.62 & 0.83 & 0.97 & 1 & 1 & 1 & 1 & 1 \\
3 & 0.42 & 0.76 & 0.96 & 1 & 1 & 1 & 1 & 1 & 1 \\
4 & 0.60 & 0.87 & 0.98 & 1 & 1 & 1 & 1 & 1 & 1 \\
5 & 0.74 & 0.95 & 0.99 & 1 & 1 & 1 & 1 & 1 & 1 \\
\hline \hline
\end{tabular}

clustering and f-SVAR model-based estimators as a function of $N$ when $K=2$. In Fig. 3, we also plots medians and MADs of squared errors of the estimated directed connectivity matrix $\left\|\widehat{\boldsymbol{\Phi}}_{\mathbf{y}}^{[j]}-\boldsymbol{\Phi}_{\mathbf{y}}^{[j]}\right\|_{F}^{2}$ for each state $j=1,2$ for increasing $N$. The medians and deviations over replications respectively indicate the accuracy (unbiasedness) and consistency of the estimator. From Fig. 22(a), both the f-SVAR estimators $\widehat{S}_{t}^{\text {SKF }}$ and $\widehat{S}_{t}^{\text {SKS }}$ perform better in regime segmentation than the K-means clustering, with substantially higher accuracy consistently for all $N$, albeit with larger deviations of estimates. The refined SKS estimates based on the entire observations are more accurate than the SKF. The RI of K-means clustering drops slowly as $N$ increases, while for both switching Kalman estimates, it tends to stabilize for high dimensions when $N \geq 20$. This may be due to the use of noisy high-dimensional TVVAR coefficient estimated from short-windowed samples in the regime partitioning, as compared to the lower-dimensional, reliably estimated subspace of factors in our approach. Another reason is the inherent limitation of the $\mathrm{K}$-means algorithm itself neglecting temporal evolution of the connectivity states, which instead can be captured by the Markov chain of the switching models.

From Fig. 2(b), it is shown that the f-SVAR subspace estimators clearly outperform the $L_{2}$-regularized VAR estimator based on K-means clustered regimes, particularly when $N$ is large relative to $T$, in terms of significantly lower estimation mean squared errors. Both methods perform comparably when $N$ is small. We can also see a rapidly growing trend of estimation errors in the K-means clustering-based $L_{2}$-estimator as $N$ increases and approaches the regime sample size. In contrast, the robustness of the proposed f-SVAR estimators in highdimensional settings is evident from the slower error rates and

Fig. 2. Effect of dimensionality $N$ on the connectivity state estimation performance of K-means clustering and f-SVAR approaches when $K=2$ and $T=200$. (a) Rand index of state segmentation. (b) MSE of dynamic connectivity matrices. Lines and error bars represent medians and median absolute deviations over 200 replications.

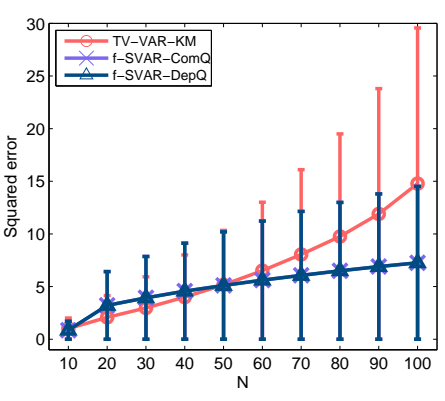

(a)

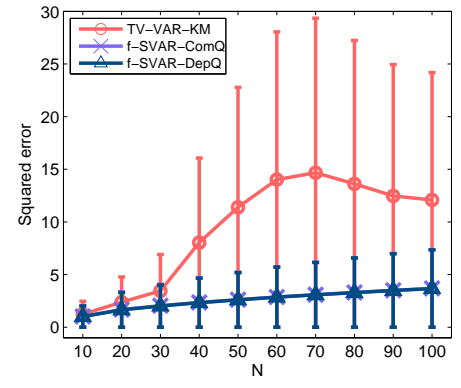

(b)
Fig. 3. Squared errors of estimated connectivity matrix at each state $j$ as a function of network dimensionality $N$. (a) $j=1$. (b) $j=2$. Lines and error bars represent medians and median absolute deviations over 200 replications.

the constancy of standard errors (indicated by the small median deviations) over the increasing dimensions. The percentage of reduction in MSE by f-SVAR-DepQ over the TV-VAR-KM achieves $60 \%$ when $N=100$ which is equal to sample size available for each regime. This improvement in MSE for the dynamic connectivity can be explained by the more accurate regime segmentation by the SKS (as shown in Fig. 22a)), and the better consistency of the factor-based estimator over the ridge estimator for high-dimensional connectivity matrix in each regime (as shown in Fig. 3). The f-VAR estimator has been already shown to improve the consistency and computational efficiency substantially under high-dimensional settings, compared to the traditional least-squares estimator [16]. Among the f-SVAR methods, the coupled estimator given optimal $r$ selected by BIC, now achieves similar performance as the decoupled estimator despite slight superiority of the later. This suggests that in this simulation the difference in directed connectivity structure between states is mostly captured by inter-dependence in the factors, where further use of distinct subspace projection $\mathbf{Q}$ for each state only gives marginal improvement.

6) Effect of Varying Number of States: We assess the scalability of our estimators in identifying increased number of connectivity states $K$ with a fixed time series length. Fig. 4 plots the results as a function of $K=2,3,4,5$ with fixed $T=200$ and $N=25(M=5, n=5)$. The f-SVAR estimators substantially outperform the K-means clustering in both measures for all $K$. From Fig. 4(a), it is interesting to see improvement in regime partitioning of all methods as $K$ 


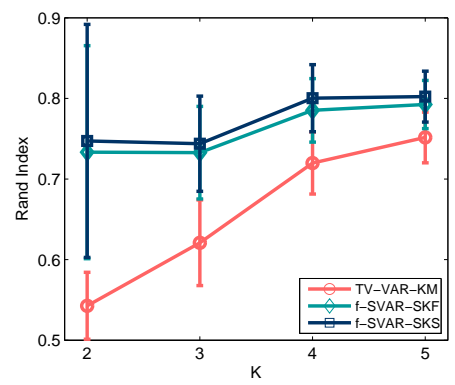

(a)

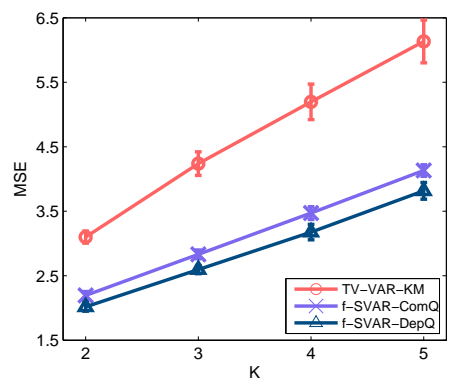

(b)

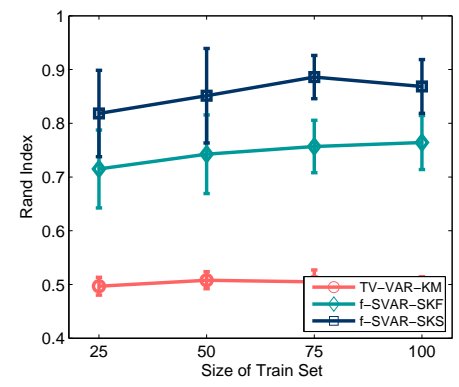

(a)

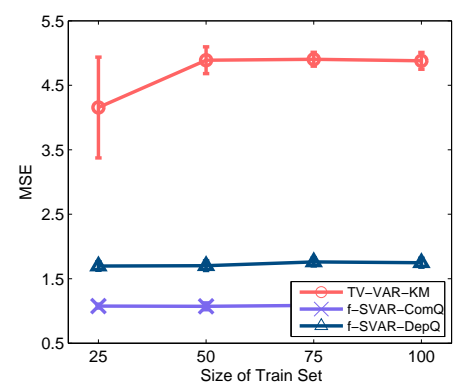

(b)

Fig. 4. Effect of number of states $K$ on the connectivity state estimation performance of K-means clustering and f-SVAR approaches when $N=25$ and $T=200$. (a) Rand index of state segmentation. (b) MSE of dynamic connectivity matrices. Lines and error bars represent medians and median absolute deviations over 200 replications.

increases. Note that, for a fixed $T$, more change points were simulated with larger $K$. This suggests all methods considered here are more efficient in tracking abrupt changes rather than smooth dynamics in connectivity. Nevertheless, the f-SVAR approach still performs much better in capturing the more stationary structures, indicated by substantially higher RIs at $K=2$ and $K=3$ than the K-means clustering. In contrast, Fig. 4(b) shows an increase in estimation errors for dynamic connectivity despite the improved regime segmentation. This is due to the poor estimation of the state-dependent connectivity graphs, given a decreased sample size $T_{B}$ available to estimate the large number of parameters in each state when $K$ increases. Again, there is slight improvement of the SKS over SKF in RI, and the decoupled f-SVAR estimator over coupled estimator in MSE.

7) Prediction of Regime Partitions: We further evaluate the performance of pre-trained f-SVAR models in predicting the regime segmentation and time-evolving graphs on an unknown testing data. We used 5-fold cross-validation, where in each fold $4 / 5$ of the $R$ generated time series replicates (with $T=100, N=25$ for $K=2$ ) were used as training set to fit a predictive model. Here the K-means clustering was applied to concatenated data of the training replicates. The f-SVAR parameters were updated in EM by aggregating information across replicates using (20)-22). The remaining $1 / 5$ data was holdout as validation set to compute the performance of the fitted model in RI and MSE. Fig. 5 plots the prediction performance against the size of training set $R$. As expected, the prediction of regime partitions and connectivity graphs by the f-SVAR estimators improves when given more training data, as shown in Fig. 5(a) and Fig. 5(b). In consistency with the estimation results in above simulations, our approach also gives better prediction for dynamic connectivity than the Kmeans clustering in both performance measures.

\section{Application to Resting-State fMRI Data}

In this section, we apply the proposed f-SVAR approach to estimating dynamic effective connectivity in high-dimensional resting-state fMRI data, characterized by abrupt transition of underlying quasi-stable brain states.

\section{A. Data Acquisition \& Preprocessing}

We studied resting-state fMRI data of 25 subjects from a dataset publicly available at [26]. Subjects were asked to

Fig. 5. Effect of the size of training set $R$ on the connectivity state prediction performance of K-means clustering and f-SVAR approaches when $N=25$, $T=100$ and $K=2$. (a) Rand index of state segmentation. (b) MSE of dynamic connectivity matrices. Lines and error bars represent medians and median absolute deviations over validation sets of $S / 5$ replications based on 5-fold cross-validation.

relax during scans. Resting state fMRI scans were acquired on a Siemens Allegra 3-Tesla scanner using a T2-weighted gradient-echo planar imaging $(\mathrm{EPI})$ sequence $(\mathrm{TR} / \mathrm{TE}=$ $2000 / 25 \mathrm{~ms}$; flip angle $=90^{\circ}$; field of view $=192 \mathrm{~mm}$; voxel size $=3 \times 3 \times 3 \mathrm{~mm}^{3}$; matrix $64 \times 64 ; 39$ slices). A time series of $T=197$ EPI volumes was collected for each scan. The data were preprocessed using the AFNI and FSL software packages, following steps in [27].

We used automated anatomical labeling (AAL) atlas to obtain an anatomical parcellation of the whole brain into 90 regions of interest (ROIs) with 45 regions in each hemisphere. In this study, we grouped these ROIs into six pre-defined resting-state system networks (RSNs) of similar anatomical and functional properties, based on the templates in [6], [28]. The considered RSNs include sub-cortical (SCN), AN: auditory (AN), sensorimotor (SMN), visual (VN), attentional (ATN) and default mode network (DMN). Refer to Supplementary material for mapping of ROIs to the corresponding RSNs. We followed the ROI abbreviations in [29].

\section{B. Results}

We analyzed the dynamic states of large-scale directed brain connectivity in the resting state. We fitted a three-state fSVAR(1) model using the EM algorithm to the resting-state ROI fMRI time series from 20 subjects (other 5 subjects were reserved to evaluate state prediction). The estimated state-time sequence and state-dependent VAR coefficient matrices were used to identify the state transitions and the high-dimensional directed dependencies within each state. Here, the decoupled SVAR subspace estimator was used. The number of factors selected by BIC for this data was $\hat{r}=14$. We set the model order $P=1$ as suggested by BIC, which is also typically used for fMRI data when $N$ is large [30]. We chose the number of connectivity clusters $K=3$ suggested by the variance ratio criterion by applying K-means clustering of TV-VAR coefficients based on concatenated data of all subjects.

Fig. 6 shows the estimated whole-brain directed connectivity matrices $\widetilde{\mathbf{\Phi}}_{\mathbf{y}}^{[j]}$ between ROIs for three distinct states. Only significant connections are shown, tested based on the asymptotic normality of the f-SVAR coefficient estimator in 23, at $\alpha=0.05$ with Bonferroni correction. As expected from simulations, the TV-VAR-KM approach produces very noisy 
estimates for the high-dimensional connectivity matrices. In contrast, our method identifies the modular structure of the resting-state networks over all states, where ROIs within a functionally relevant network tend to be densely connected, but sparsely connected between different networks (indicated by intense red color in the diagonal blocks), particularly pronounced in VN, DMN and SMN. This characteristic has been reported in previous studies of static fMRI functional connectivity, e.g. [31]. In consistency with findings in dynamic functional connectivity states [3], [6], our results also show the distinct large-scale connectivity patterns across different brain states in terms of variability in both the strength and sign of the connectivity and the network modularity. More interestingly, our method further reveals state-related differences in the directionality of the connections, not reported in other similar studies. These difference are evident from the asymmetry of the estimated VAR coefficient matrices. It is shown that the states are also differentiated by the ROI-wise connectivity for both between-networks and within-networks (quantified respectively by off-diagonal and diagonal blocks).

Fig. 7 shows the within-network connectivity graphs for four selected RSNs, extracted from the f-SVAR matrices in Fig. 6. We describe some apparent differences in the effective connectivity patterns between states. Generally, we observe stronger and denser connections between ROIs in states 1 and 3 than in state 2, for all four RSNs. For the sensorimotor networks, state 3 exhibits the highest directed connectivity between central regions in the primary motor cortex, which also receive uni-directional influences from the supplementary motor area (SMA) not detected in states 1 and 2. We also found interactions between the SMA and both the superior parietal nodes (SPG.L and SPG.R) exclusively for state 1. The visual networks have the densest within-network connections among the RSNs, observed consistently for all states. For attentional networks, our method identified the lateral frontalparietal network (similar to ventral attention network [32]) between regions e.g. middle frontal gyrus and inferior parietal gyrus in all states, with the strongest connections occurred in state 3. Moreover, we found denser directed information flows across both left and right hemispheres in states 1 and 3, compared to state 2 . It is interesting that the cross-hemisphere connections between frontal regions detected in states 1 and 3 were completely absent in state 2 . In the default mode networks, we see high connectivity between ROIs related to posterior cingulate cortex (PCC)/precuneus, medial prefrontal cortex and the left and right inferior parietal lobule, with the greatest strength in state 3 . In line with numerous studies (e.g., [33]), the PCC is correctly identified as a major hub of the DMN strongly connected with other regions, as particularly pronounced in state 1.

Fig. 8 shows the SKS-estimated temporal dynamics $\widehat{S}_{t}^{\text {SKS }}$ associated with the connectivity states in Fig. 6 for each of the 25 subjects. Note that results for subjects 21-25 are predicted from the f-SVAR model fitted on subjects 1-20. The results suggest changes in effective connectivity states over time with substantial inter-subject variability in the dynamic patterns. Nevertheless, the connectivity states reoccur over time and shared across subjects. It also exhibits slow dynamics, where the connectivity tends to be assigned to single discrete states for long periods, with occasional fast switching between states. Moreover, the degree of the non-stationarity differs between subjects, from the rapid transitions between states (e.g. subjects 5-9) to almost time-constant connectivity remained in certain states (e.g. 16-18), predominantly state 2 (blue). Note that state 3 (green) shows enhanced connectivity for all RSNs, but has the lowest temporal occupancy in most subjects.

Fig. 9 shows the estimates for subject 9 for detailed illustration. In our model, the changes between the connectivity states of the high-dimensional fMRI signals in Fig. 8 is characterized in the few latent factor time series $\widehat{\mathbf{f}}_{t}$ derived from $\mathbb{E}\left(\mathbf{F}_{t} \mid \mathbf{y}_{1: T}\right)$ (see Fig. 9. (a) for the first five estimated components). The temporal instability in $\widehat{\mathbf{f}}_{t}$, as indicated by the change-point estimates from SKS $\widehat{S}_{t}^{\text {SKS }}$ (dotted lines), is essentially reflected in the structural breaks in its correlation structure specified in the time-evolving factor VAR coefficient matrices $\widehat{\boldsymbol{\Phi}}_{\mathbf{f}}\left[\widehat{S}_{t}\right]$ in Fig. 9(b)(bottom). For example, the relation between the peak of the 1-st factor and the trough of the 4-th factor signal centered around $T=140$, is quantified by the negative correlation (in blue color). Compared to the much smaller number of factor-based coefficients $(14 \times 14=196$ entries $)$, the high-dimensional sliding-windowed TV-VAR coefficients $(96 \times 96=9216$ entries $)$ fitted directly to the observed signals, are unable to accurately identify the regime changes, due to poor estimates limited by window size (despite a large window of 20 samples was used). Moreover, it produces a overly smooth regime partition in the K-means clustering, which neglects the detailed dynamic structures identified by the fSVAR estimator as illustrated in Fig. 9(c). Besides, the SKS refines the state estimates by SKF, smoothing the spurious spikes and producing more stable regimes.

\section{DISCUSSION}

We developed a generalization of VAR model for identifying dynamic effective connectivity states with a large number of brain regions from fMRI data, based on a regime-switching factor model. The proposed approach first characterizes the high-dimensional fMRI data via a small number of factors for dimension reduction using a factor model in the observation space, and then performs connectivity regime segmentation in this low-dimensional latent factor subspace. By specifying the factor dynamics as a Markov-switching VAR process with a state-space formulation, it enables a reliable and efficient detection of change-points of connectivity states using the Kalman smoothing and EM algorithm, and estimation of high-dimensional connectivity matrix for each state based on subspace projection via a novel f-SVAR model.

The regime-switching models have been proven more effective than sliding-window approach in handling changes at different time scales [15]. It also differs from the classical piecewise constant processes (e.g., time-varying VAR processes [11]) in a sense that it permits recurring connectivity regimes where future blocks could be related to past blocks if both were indexed by the same state. Information from different time blocks of the same regime can be pooled together to produce more accurate estimates. The f-SVAR extends this to identify state dynamics in large-sized brain networks. The 
State 1
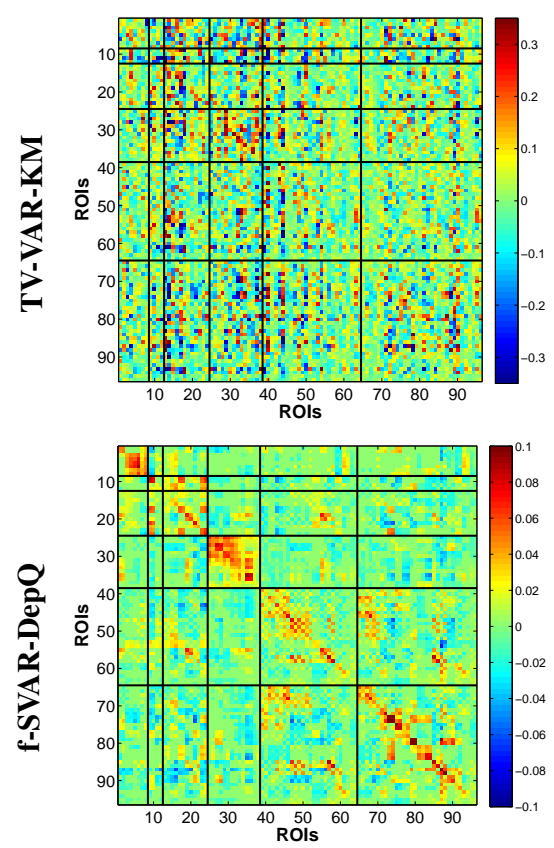

State 2
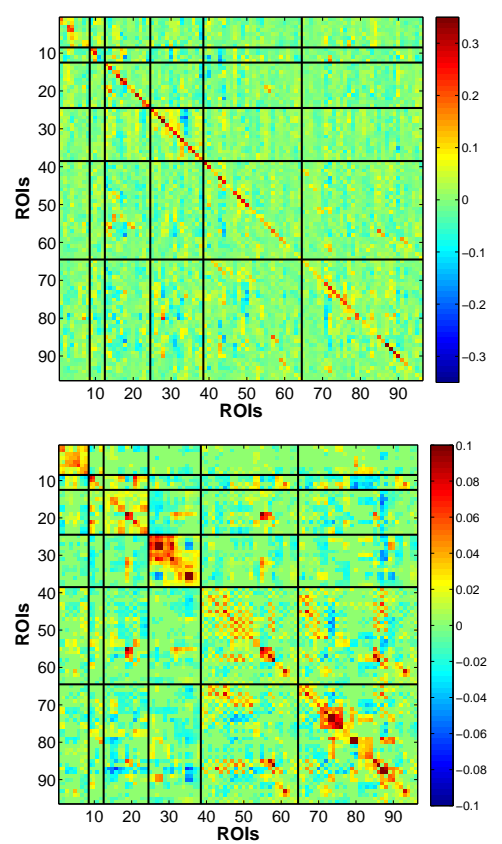

State 3
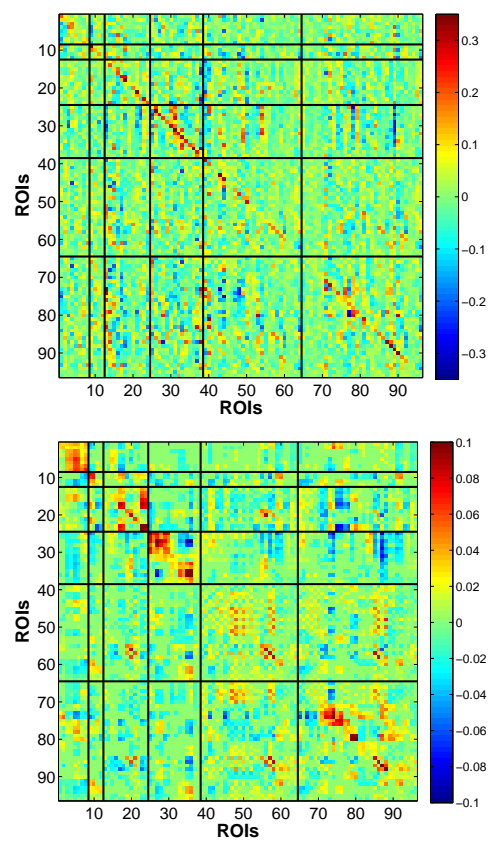

Fig. 6. Effective connectivity states in the resting-state fMRI data estimated based on 20 subjects. Comparison of VAR coefficient matrices which represent the whole-brain connectivity between ROIs at each state, obtained by the K-means clustering (Top) and f-SVAR approaches (Bottom). The 90 brain ROIs are grouped with overlapping into six RSNs: sub-cortical (SCN), auditory (AN), sensorimotor (SMN), visual (VN), attentional (ATN) and default mode network (DMN). The partitions are indicated by lines. The AR coefficient entries of the f-SVAR model are significantly different from zero at level $\alpha=0.05$ with Bonferroni correction for multiple testings.
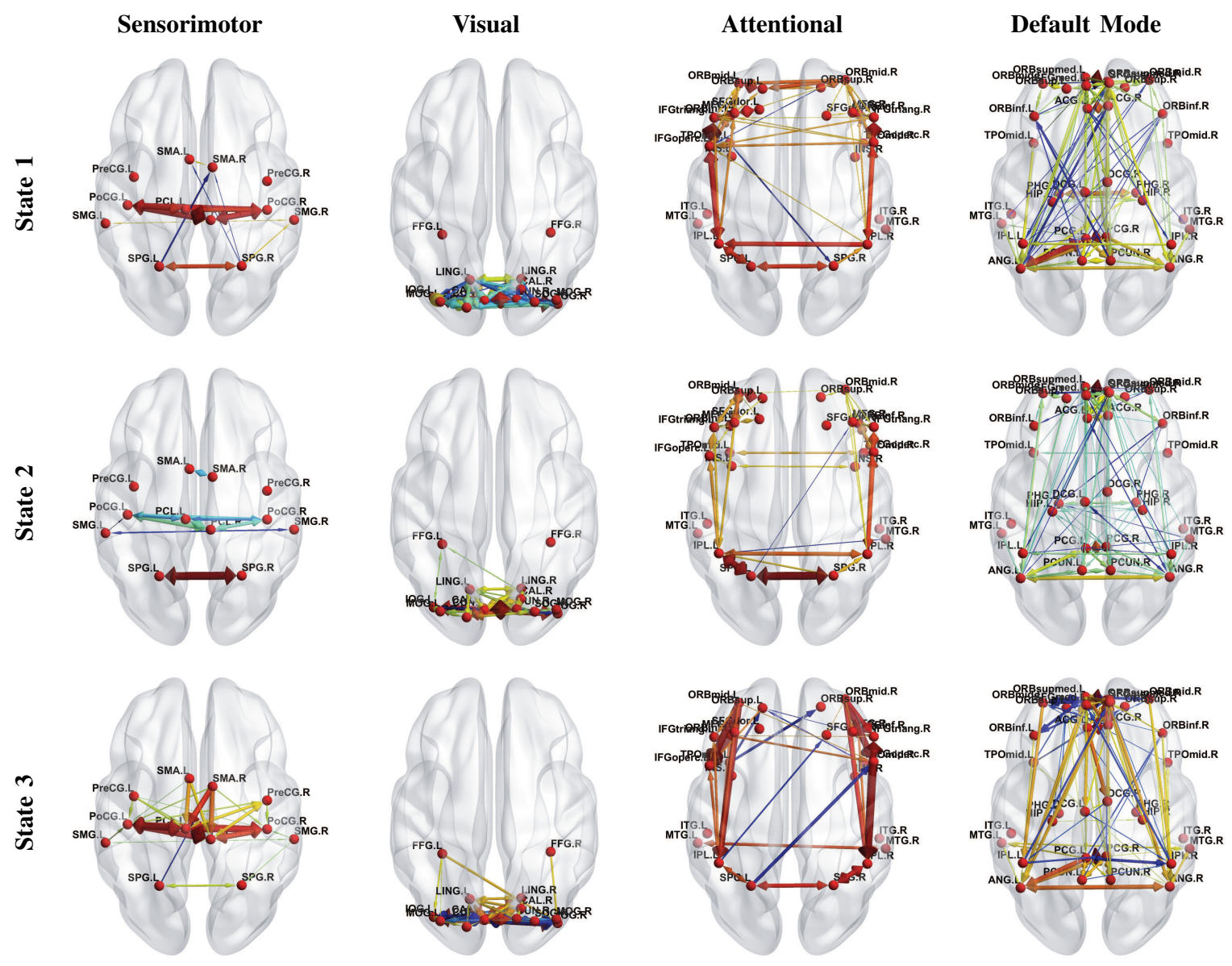

Fig. 7. Topological representations of within-network directed connectivity for four resting-state networks (RSNs) identified by the factor SVAR approach, show distinct large-scale connectivity patterns across three states. Edges represents strong connections with absolute values of AR coefficient greater than a threshold of 0.03 , and arrows indicate the directionality of the connections. 


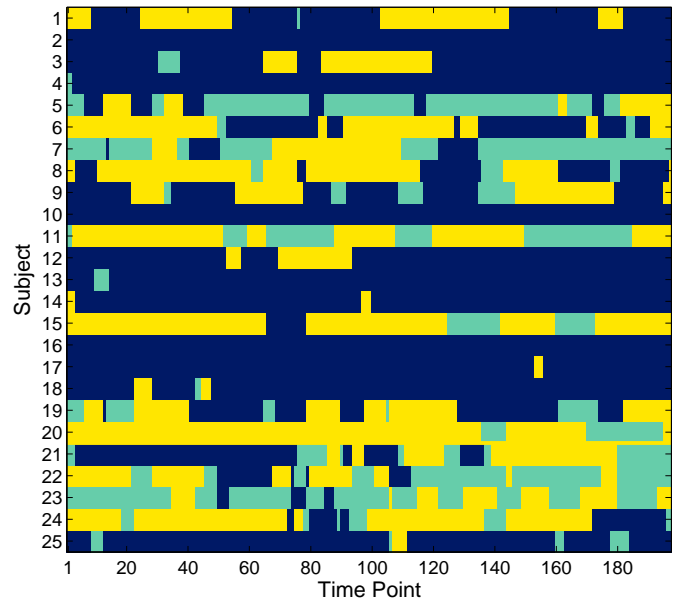

Fig. 8. Estimated temporal dynamics of effective connectivity states in fMRI data across 25 subjects during the resting state. State 1: yellow, State 2: blue, State 3: green. The state sequences for subjects 1-20 were estimated by SKS based on a f-SVAR model fitted on the same 20 subjects, and those for subjects 21-25 were predicted based on the 20-subjects model

use of VAR specification further allows us to examine statedriven changes in the directionality of the connections, which is not addressed in earlier studies of dynamic functional (undirectional) connectivity. A related work [34] used HMM with VAR as observation model to identify dynamic directed connectivity states in electrophysiological data. Our proposed model is more general allowing each Markov state to drive a distinct VAR state-space model, and more robust to highdimensions by projecting the temporal dynamics in the factor space. A switching factor analysis with Bayesian inference was proposed recently by [35] which however does not account for the temporal dependence in the latent factors, and hence is only applicable to time-varying functional networks.

Simulation results show superiority of our approach over the K-means clustering of TV-VAR coefficients, giving more accurate estimation of the dynamic states and the withinstate connectivity graph, particularly in the high-dimensional settings. Applied to resting-state fMRI data, the proposed fSVAR estimator confirms previous findings of non-stationary, re-occurring brain states during rest, and state-dependent modulation of large-scale connectivity patterns and modular structure. Furthermore, we produced new evidence for acrossstate difference in both the strength and directionality of directed information flows within resting-state networks.

There are potential limitations of our proposed approach. First, the low-rank assumption of factor modeling might not be appropriate for some connectivity structures. Future work can incorporate sparsity based on the idea of low-rank plus sparse modeling [36] to explain a broader class of network topologies. Secondly, despite the ability to handle multisubject estimation, the current framework does not quantify the variations in dynamic connectivity patterns across subjects. It can be extended to mixed-effect modeling [37] to account for inter-subject variability. Another weakness is the use of Markov chain which may produce unrealistically rapid switching dynamics with non-Markovian behavior in the data. This could be mitigated by using semi-Markov models which allows explicit control over state durations to encourage temporal homogeneity (smoothness). Moreover, our method

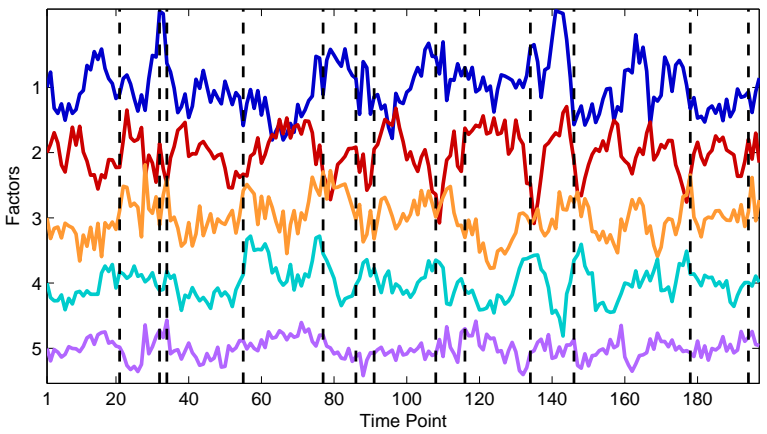

(a)
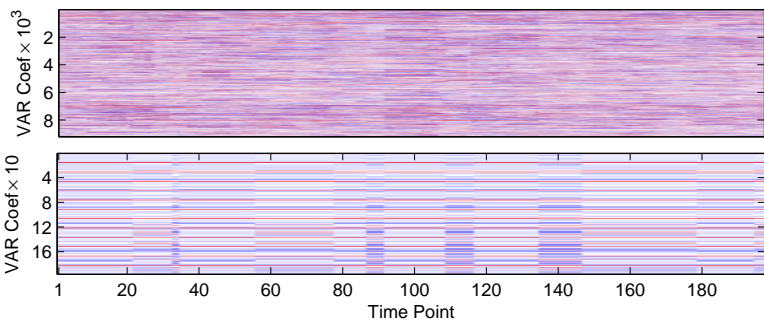

(b)

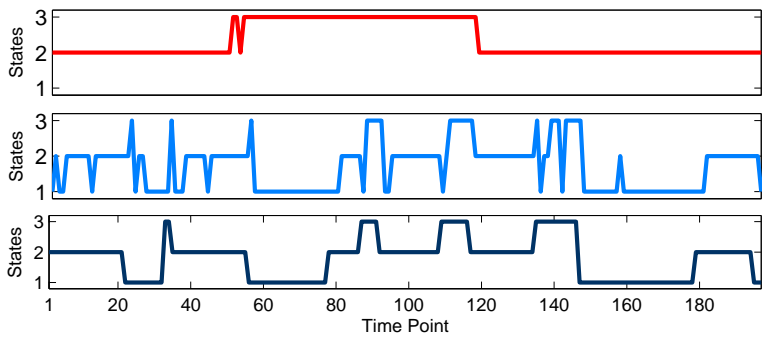

(c)

Fig. 9. Tracking the temporal changes in effective connectivity states in a real fMRI data for subject 9. (a) Estimated first 5 factor time series using the f-SVAR model. (b) Estimated time-evolving VAR coefficients (vectorized) in observation space by sliding windows (Top) and in factor space $\widehat{\boldsymbol{\Phi}}_{\mathbf{f}}^{\left[\widehat{S}_{t}\right]}$ by EM algorithm (Bottom). (c) Estimated state sequence by the K-means clustering (red), SKF (light blue) and SKS (dark blue). Dotted lines indicate regime segmentation by SKS.

may be computationally ineffective for networks with ultrahigh dimensions in voxel-wise full-brain analysis. One strategy is to apply the f-SVAR model to voxel data in parcellated ROIs as in [38], and then estimate within- and betweenROI dynamic connectivity based on these ROI-specific factors. Another possible extension is to permit regime-switching in both the factor loadings and factor dynamics to detect changes in brain community structure [39]. Besides PCA, one could also investigate alternative subspace projection methods for dimension reduction such as independent component analysis (ICA) in the current framework. Our method is also applicable to task fMRI data to explore association between transitions of latent brain states with the presentation of different stimuli.

The Matlab code implementing the f-SVAR method with its documentation is freely available for download from GitHub https://github.com/CheeMingTing/f-SVAR

\section{APPENDIX}

In Proposition 1, we present the limiting distribution of the decoupled estimator of f-SVAR coefficient matrix $\widetilde{\boldsymbol{\Phi}}_{\mathbf{y}}^{[j]}$ for each state $j$. It directly follows from results derived in [16] (Theorem 2), the consistency of $\widetilde{\mathbf{f}}_{t}^{[j]}$ and $\widetilde{\mathbf{Q}}^{[j]}$ established in 
[20], [21], and the consistency of the estimated state sequence for regime partitioning, i.e. $T^{-1} \sum_{t=1}^{T}\left|\widehat{S}_{t}-S_{t}\right| \rightarrow 0$. For ease of exposition, we focus on $\operatorname{VAR}(1)$ without loss of generality.

Proposition 1 (Asymptotic Distribution of $\widetilde{\mathbf{\Phi}}_{\mathbf{y}}^{[j]}$ ): Suppose Assumptions A-D in [20] holds. Let $T_{j}$ and $T=\sum_{t=1}^{T} T_{j}$ be the sample size for regime $j$ and entire signal respectively. The vectorized decoupled f-SVAR estimator $\widetilde{\mathbf{b}}^{[j]}=\operatorname{vec}\left(\widetilde{\boldsymbol{\Phi}}_{\mathbf{y}}^{[j]}\right)=$ $\operatorname{vec}\left(\widetilde{\mathbf{Q}}^{[j]} \widetilde{\boldsymbol{\Phi}}_{\mathbf{f}}^{[j]} \widetilde{\mathbf{Q}}^{[j]^{\prime}}\right)$ for each $j$ is consistent and asymptotically normal, as $T_{j}, T \rightarrow \infty$ with $T_{j} / T \rightarrow c_{j} \in(0,1)$

$$
\sqrt{T_{j}}\left(\widetilde{\mathbf{b}}^{[j]}-\mathbf{b}^{[j]}\right) \stackrel{D}{\rightarrow} N\left(\mathbf{0}, \mathbf{G}^{[j]}\right)
$$

where $\mathbf{G}^{[j]}=\left(\mathbf{Q}^{[j]} \boldsymbol{\Sigma}_{\boldsymbol{\eta}}^{[j]} \mathbf{Q}^{[j]^{\prime}}\right) \otimes\left(\mathbf{Q}^{[j]} \boldsymbol{\Gamma}_{\mathbf{f}}^{[j]} \mathbf{Q}^{[j]^{\prime}}\right)$ with $\boldsymbol{\Gamma}_{\mathbf{f}}^{[j]}=$ $\operatorname{Cov}\left(\mathbf{f}_{t}^{[j]}\right)$ and $\otimes$ denotes the Kronecker product.

We compute the covariance of the estimator $\widehat{\mathbf{G}}^{[j]}$ by plugging in the PCA estimates. Based on this, we test the significance of each f-SVAR coefficient in $\widetilde{\mathbf{b}}^{[j]}$ for being different from zero, with $H_{0}: b_{k}^{j}=0$ against $H_{1}: b_{k}^{j} \neq 0$, where $b_{k}^{j}$ is $k$-th element of $\mathbf{b}^{[j]}$. The test statistic is defined by $t_{k}=\widehat{b}_{k}^{j} / \sqrt{\widehat{\mathbf{G}}_{k k}^{[j]} / T_{j}} \sim N(0,1)$ when $T_{j}$ is sufficiently large, where $\widehat{\mathbf{G}}_{k k}^{[j]}$ is $k$-th diagonal entry of $\widehat{\mathbf{G}}^{[j]}$. A coefficient is significant if the $p$-value $<\alpha / D$ with $\alpha$ the significance level and $D=N^{2}$ the number of tested coefficients, implying corrections for multiple testing by Bonferroni method.

\section{REFERENCES}

[1] B. Cassidy, C. Rae, and V. Solo, "Brain activity: Connectivity, sparsity, and mutual information," IEEE Trans. Med. Imag., vol. 34, no. 4, pp. 846-860, 2015.

[2] W. Liao, D. Marinazzo, Z. Pan, Q. Gong, and H. Chen, "Kernel Granger causality mapping effective connectivity on fMRI data," IEEE Trans. Med. Imag., vol. 28, no. 11, pp. 1825-1835, 2009.

[3] R. M. Hutchison et al., "Dynamic functional connectivity: Promise, issues, and interpretations," NeuroImage, vol. 80, pp. 360-378, 2013.

[4] V. D. Calhoun, R. Miller, G. Pearlson, and T. Adalı, "The chronnectome: Time-varying connectivity networks as the next frontier in fMRI data discovery," Neuron, vol. 84, no. 2, pp. 262-274, 2014.

[5] C. Chang and G. H. Glover, "Time-frequency dynamics of resting-state brain connectivity measured with fMRI," NeuroImage, vol. 50, no. 1, pp. 81-98, 2010.

[6] E. A. Allen, E. Damaraju, S. M. Plis, E. B. Erhardt, T. Eichele, and V. D. Calhoun, "Tracking whole-brain connectivity dynamics in the resting state," Cerebral Cortex, p. bhs352, 2012.

[7] R. M. Hutchison, T. Womelsdorf, J. S. Gati, S. Everling, and R. S. Menon, "Resting-state networks show dynamic functional connectivity in awake humans and anesthetized macaques," Hum Brain Mapp., vol. 34, no. 9, pp. 2154-2177, 2013.

[8] A. Zalesky, A. Fornito, L. Cocchi, L. L. Gollo, and M. Breakspear, "Time-resolved resting-state brain networks," Proc. Natl. Acad. Sci. U.S.A., vol. 111, no. 28, pp. 10341-10346, 2014.

[9] M. A. Lindquist, Y. Xu, M. B. Nebel, and B. S. Caffo, "Evaluating dynamic bivariate correlations in resting-state fMRI: A comparison study and a new approach," NeuroImage, vol. 101, pp. 531-546, 2014.

[10] M. Havlicek, J. Jan, M. Brazdil, and V. D. Calhoun, "Dynamic Granger causality based on Kalman filter for evaluation of functional network connectivity in fMRI data," NeuroImage, vol. 53, no. 1, pp. 65-77, 2010.

[11] S. B. Samdin, C.-M. Ting, Sh-H. Salleh, M. Hamedi, and A. M. Noor, "Identifying dynamic effective connectivity states in fMRI based on time-varying vector autoregressive models," in Int. Conf. Innovation in Biomedical Eng. and Life Sciences. Springer, 2015, pp. 243-247.

[12] I. Cribben, R. Haraldsdottir, L. Y. Atlas, T. D. Wager, and M. A Lindquist, "Dynamic connectivity regression: Determining state-related changes in brain connectivity," NeuroImage, vol. 61, no. 4, pp. 907-20, 2012.

[13] A. P. Baker, M. J. Brookes, I. Rezek, S. M. Smith, T. Behrens, P. J. P. Smith, and M. Woolrich, "Fast transient networks in spontaneous human brain activity," eLife, vol. 3, no. 3, pp. 1-18, 2014.
[14] S.-O. Jeong, C. Pae, and H.-J. Park, "Connectivity-based change point detection for large-size functional networks," NeuroImage, vol. 143, pp. 353-363, 2016.

[15] S. B. Samdin, C.-M. Ting, H. Ombao, and Sh-H. Salleh, "A unified estimation framework for state-related changes in effective brain connectivity," IEEE Trans. Biomed. Eng., vol. 64, no. 4, pp. 844-858, 2017.

[16] C.-M. Ting, A.-K. Seghouane, S.-H. Salleh, and A. M. Noor, "Estimating effective connectivity from fMRI data using factor-based subspace autoregressive models," IEEE Sig. Process. Lett., vol. 22, no. 6, pp. 757-761, 2014.

[17] H.-M. Krolzig, Markov-Switching Vector Autoregressions: Modelling, Statistical Inference, and Application to Business Cycle Analysis, vol. 454, Springer Science \& Business Media, 2013.

[18] C.-J. Kim, "Dynamic linear models with Markov-switching," $J$. Econometrics, vol. 60, no. 1-2, pp. 1-22, 1994.

[19] J. Bai and S. Ng, "Determining the number of factors in approximate factor models," Econometrica, vol. 70, no. 1, pp. 191-221, 2002.

[20] J. Bai, "Inferential theory for factor models of large dimensions," Econometrica, vol. 71, pp. 135-171, 2003.

[21] J. H. Stock and M. W. Watson, "Forecasting using principal components from a large number of predictors," J. Amer. Statist. Assoc., vol. 97, pp. $1167-1197,2002$.

[22] K. P. Murphy, "Switching Kalman filters," Tech. Rep., UC Berkeley, 1998.

[23] R. P. Monti, P. Hellyer, D. Sharp, R. Leech, C. Anagnostopoulos, and G. Montana, "Estimating time-varying brain connectivity networks from functional MRI time series," NeuroImage, vol. 103, pp. 427-43, 2014.

[24] D. Korobilis, "VAR forecasting using Bayesian variable selection," $J$. Appl. Econometrics, vol. 28, no. 2, pp. 204-230, 2013.

[25] W. M. Rand, "Objective criteria for the evaluation of clustering methods," J. Amer. Statist. Assoc., vol. 66, no. 336, pp. 846-850, 1971.

[26] NITRC, "NYU CSC TestRetest,"'https://www.nitrc.org/projects/nyu_trt

[27] M. Fiecas, H. Ombao, D. van Lunen, R. Baumgartner, A. Coimbra, and D. Feng, "Quantifying temporal correlations: A test-retest evaluation of functional connectivity in resting-state fMRI," NeuroImage, vol. 65, pp. 231-241, 2013

[28] R. Li, K. Cheni, A. S. Fleishei, E. M. Reimani, L. Yao, and X. Wu, "Large-scale directional connections among multi resting-state neural networks in human brain: A functional MRI and Bayesian network modeling study," NeuroImage, vol. 56, pp. 1035-1042, 2011.

[29] R. Salvador, J. Suckling, M. R. Coleman, J. D. Pickard, D. Menon, and E. Bullmore, "Neurophysiological architecture of functional magnetic resonance images of human brain," Cereb. Cortex, vol. 15, pp. 13321342, 2005.

[30] P. A. Valdés-Sosa et al., "Estimating brain functional connectivity with sparse multivariate autoregression," Philos. Trans. R. Soc. B, vol. 360, no. 1457 , pp. 969-981, 2005.

[31] L. Ferrarini et al., "Hierarchical functional modularity in the restingstate human brain," Hum Brain Mapp., vol. 30, pp. 2220-2231, 2009.

[32] J. Vincent, I. Kahn, A. Snyder, M. Raichle, and R. Buckner, "Evidence for a frontoparietal control system revealed by intrinsic functional connectivity," J. Neurophysiol., vol. 100, pp. 3328-3342, 2008.

[33] P. Fransson and P. Marrelec, "The precuneus/posterior cingulate cortex plays a pivotal role in the default mode network: Evidence from a partial correlation network analysis," NeuroImage, vol. 42, pp. 1178-1184, 2008.

[34] D. Vidaurre, A. J. Quinn, A. P. Baker, D. Dupret, A. Tejero-Cantero, and M. W. Woolrich, "Spectrally resolved fast transient brain states in electrophysiological data," NeuroImage, vol. 126, pp. 81-95, 2016.

[35] J. Taghia, S. Ryali, T. Chen, K. Supekar, W. Cai, and V. Menon, "Bayesian switching factor analysis for estimating time-varying functional connectivity in fMRI," NeuroImage, vol. 155, pp. 271-290, 2017.

[36] J. Fan et al., "Large covariance estimation by thresholding principal orthogonal complements," J. Royal Stat. Society: Series B, vol. 75, no. 4, pp. 603-680, 2013.

[37] B. Ng, M. J. McKeown, and R. Abugharbieh, "Group replicator dynamics: A novel group-wise evolutionary approach for sparse brain network detection," IEEE Trans. Med. Imag., vol. 31, no. 3, pp. 576585, 2012.

[38] Y. Wang, C.-M. Ting, and H. Ombao, "Modeling effective connectivity in high-dimensional cortical source signals," IEEE J. Sel. Topics Signal Process, vol. 10, no. 7, pp. 1315-1325, 2016.

[39] H. Chen, K. Li, D. Zhu, X. Jiang, Y. Yuan, P. Lv, T. Zhang, L. Guo, D. Shen, and T. Liu, "Inferring group-wise consistent multimodal brain networks via multi-view spectral clustering," IEEE Trans. Med. Imag., vol. 32, no. 9, pp. 1576-1586, 2013. 